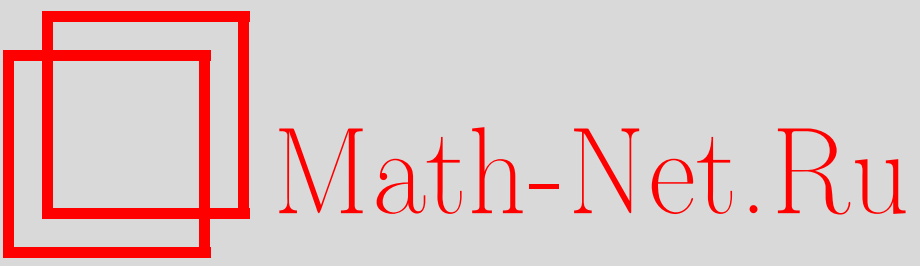

К. У. Хубиев, Краевая задача для нагруженного гиперболо-параболического уравнения с вырождением порядка, Итоги науки и техн. Сер. Соврем. мат. и ее прил. Темат. обз., 2019, том 167, 112-116

DOI: https://doi.org/10.36535/0233-6723-2019-167-112-116

Использование Общероссийского математического портала Math-Net.Ru подразумевает, что вы прочитали и согласны с пользовательским соглашением

http://www.mathnet.ru/rus/agreement

Параметры загрузки:

IP : 54.237 .206 .68

26 апреля 2023 г., 13:12:17 


\title{
КРАЕВАЯ ЗАДАЧА ДЛЯ НАГРУЖЕННОГО ГИПЕРБОЛО-ПАРАБОЛИЧЕСКОГО УРАВНЕНИЯ С ВЫРОЖДЕНИЕМ ПОРЯДКА
}

\author{
(c) 2019 г. $\quad$ К. У. ХУБИЕВ
}

\begin{abstract}
АннотАция. В работе исследуется краевая задача с разрывными условиями сопряжения на линии изменения типа для модельного уравнения смешанного гиперболо-параболического типа с вырождением порядка в области его гиперболичности. В параболической области уравнение представляет собой уравнение дробной диффузии, в гиперболической - нагруженное односкоростное уравнение переноса. Доказана теорема единственности и существования решения, выписано явное решение задачи в параболической и гиперболической областях.
\end{abstract}

Ключевые слова: краевая задача, нагруженное уравнение, уравнение смешанного типа, гиперболо-параболическое уравнение, уравнение дробной диффузии, уравнение переноса.

\section{BOUNDARY-VALUE PROBLEM \\ FOR A LOADED HYPERBOLIC-PARABOLIC EQUATION WITH DEGENERATION OF ORDER}

\author{
(c) 2019 K. U. KHUBIEV
}

\begin{abstract}
In this paper, we study a boundary-value problem with discontinuous conjugation conditions on the line of type changing for a model equation of mixed hyperbolic-parabolic type with order degeneration in the hyperbolicity domain. In the parabolic domain, the equation is the fractional diffusion equation, whereas in the hyperbolic domain it is the loaded one-speed transport equation. We prove the uniqueness and existence theorem and propose an explicit solution of the problem in the parabolic and hyperbolic domains.
\end{abstract}

Keywords and phrases: boundary-value problem, loaded equation, mixed-type equation, hyperbolicparabolic equation, fractional diffusion equation, transport equation.

AMS Subject Classification: 35M10, 35M12

1. Введение и постановка задачи. Рассмотрим уравнение

$$
\begin{cases}u_{x x}-D_{0 y}^{\alpha} u=0, & y>0, \\ u_{x}+u_{y}+c u+\lambda u\left(x_{0}, y\right)=0, & y<0,\end{cases}
$$

в области $\Omega=\Omega^{+} \cup \Omega^{-}$, где $\Omega^{+}=\{(x, y): 0<x<r, y>0\}, \Omega^{-}$- область, ограниченная характеристиками $x-y=0, x-y=r$ уравнения (1) и отрезком прямой $y=x_{0}-r$ при $y<0 ; x_{0} \in\left[0, r\left[, \lambda, c=\right.\right.$ const, $0<\alpha<1, D_{a x}^{\alpha}$ - оператор дробного интегро-дифференцирования 
Римана-Лиувилля порядка $\alpha$ с началом в точке $a$ и с концом в точке $x$ (см. $[6$, с. 9]):

$$
D_{a x}^{\alpha} \varphi(t)= \begin{cases}\frac{\operatorname{sign}(x-a)}{\Gamma(-\alpha)} \int_{a}^{x} \frac{\varphi(t) d t}{|x-t|^{\alpha+1}}, & \alpha<0, \\ \varphi(x), & \alpha=0, \\ \operatorname{sign}^{[\alpha]+1}(x-a) \frac{\partial^{[\alpha]+1}}{\partial x^{[\alpha]+1}} D_{a x}^{\alpha-[\alpha]-1} \varphi(t), & \alpha>0,\end{cases}
$$

где $[\alpha]$ - целая часть числа $\alpha$, удовлетворяющая неравенству $[\alpha] \leqslant \alpha<[\alpha]+1, \Gamma(\alpha)$ - гаммафункция Эйлера (см. [5, с. 25]).

При $y>0$ уравнение (1) является уравнением дробной диффузии (см. [8]), а при $y<0-$ нагруженным односкоростным уравнением переноса; в математической биологии при $\lambda=0$ оно известно как уравнение МакКендрика (см. [5, с. 179]).

Краевые задачи для нагруженных уравнений с частными производными исследовались в работах многих авторов (см. например, $[4,7]$ и библиографию в этих работах). В настоящее время теория краевых задач для нагруженных уравнений смешанного типа продолжает интенсивно развиваться. Краевые задачи для модельных нагруженных уравнений смешанного гиперболопараболического типа и эллиптико-гиперболического типа с нагруженным слагаемым $\lambda u(x, 0)$ и вырождением порядка в области его гиперболичности как в ограниченной, так и в неограниченной областях исследованы в [7]. В этой же монографии исследованы внутренние краевые задачи для уравнений смешанного гиперболо-параболического типа с характеристической и нехарактеристической нагрузками и их связь с аналогом задачи Трикоми (см. [7, с. 165]).

В [1] исследован эффект влияния «нагрузки» $\lambda u_{x}(x, 0)+\mu u_{y}(0, y)$ на корректную постановку начально-краевых задач для существенно нагруженного дифференциального уравнения в частных производных первого порядка. В [18] для нагруженного уравнения МакКендрика-фон Ферстера исследована задача Коши с данными на нехарактеристических и характеристических линиях.

Отметим также работы $[2,3,9-14,16,17,19-22]$, в которых исследуются локальные и нелокальные краевые задачи для уравнений смешанного типа второго и третьего порядков, содержащих оператор с дробной производной.

Краевая задача для нагруженного уравнения гиперболо-параболического типа с вырождением порядка в области его гиперболичности для уравнения, которое в $\Omega^{+}$имело вид $u_{x x}-u_{y}+$ $\lambda_{1} u\left(x_{1}, y\right)=0$, была исследована в [15]. В настоящей работе для уравнения (1) исследуется краевая задача в области $\Omega$.

Обозначим через $J$ интервал $0<x<r$ прямой $y=0$. Под регулярным решением уравнения (1) в области $\Omega$ будем понимать функцию $u=u(x, y)$ из класса

$$
y^{1-\alpha} u \in C\left(\bar{\Omega}^{+}\right), \quad y^{1-\alpha}\left(y^{1-\alpha} u\right)_{y} \in C\left(\Omega^{+} \cup J\right), \quad u_{x x} \in C\left(\Omega^{+}\right), \quad u \in C\left(\bar{\Omega}^{-}\right) \cap C^{1}\left(\Omega^{-}\right),
$$

удовлетворяющую уравнению (1) в $\Omega^{+} \cup \Omega^{-}$.

Для уравнения (1) исследуется следующая задача.

Задача 1. Найти регулярное в области $\Omega$ решение $u(x, y)$ уравнения $(1)$, удовлетворяющее условиям

$$
u(0, y)=\varphi_{0}(y), \quad u(r, y)=\varphi_{r}(y), \quad y>0
$$

кроме того, на линии $y=0$ выполняются условия сопряжения:

$$
\begin{gathered}
\lim _{y \rightarrow 0+} y^{1-\alpha} u(x, y)=\lim _{y \rightarrow 0-} u(x, y), \\
\lim _{y \rightarrow 0+} y^{1-\alpha}\left(y^{1-\alpha} u(x, y)\right)_{y}=\lim _{y \rightarrow 0-} u_{y}(x, y),
\end{gathered}
$$

где $\varphi_{0}(y), \varphi_{r}(y)$ - заданные функции.

Здесь надо отметить, что при $\lambda=0$, т.е. в гиперболической части области, задается уравнение без нагрузки, или же нагруженное слагаемое имеет вид $\lambda u(x, 0)$, решение можно найти в полуполосе $D^{-}$, ограниченной характеристиками $x-y=0, x-y=r$ уравнения (1) и отрезком 
$[0, r]$ прямой $y=0$. В случае уравнения (1) область будет ограничивается еще и отрезком прямой $y=x_{0}-r$.

2. Основные результаты. Найдем ссоотношения между $u(x, 0)$ и $u_{y}(x, 0)$ на линии изменения типа. Предположим, что существует решение $u(x, y)$ задачи $(1)-(4)$. Введем обозначения

$$
\begin{array}{lll}
\tau(x)=\lim _{y \rightarrow 0+} y^{1-\alpha} u(x, y), & x \in \bar{J}, \\
\nu(x)=\lim _{y \rightarrow 0+} y^{1-\alpha}\left(y^{1-\alpha} u(x, y)\right)_{y}, & x \in J,
\end{array}
$$

а из условий задачи (2) и (3) получим

$$
\tau(0)=\lim _{y \rightarrow 0+} y^{1-\alpha} \varphi_{0}(y)=\widetilde{\varphi}_{0}, \quad \tau(r)=\lim _{y \rightarrow 0+} y^{1-\alpha} \varphi_{r}(y)=\widetilde{\varphi}_{r} .
$$

В [2, с. 48] функциональное соотношение между $\tau(x)$ и $\nu(x)$ для уравнения $(1)$, принесенное из параболической области $\Omega^{+}$, получено в виде

$$
\nu(x)=\frac{1}{\Gamma(\alpha+1)} \tau^{\prime \prime}(x)
$$

Переходя к пределу при $y \rightarrow-0$, из $\Omega_{2}$ получим

$$
\tau^{\prime}(x)+\nu(x)+c \tau(x)+\lambda \tau\left(x_{0}\right)=0 .
$$

Из $(7),(8)$ получаем нагруженное обыкновенное дифференциальное уравнение второго порядка:

$$
\tau^{\prime \prime}(x)+\Gamma(\alpha+1) \tau^{\prime}(x)+c \Gamma(\alpha+1) \tau(x)=-\lambda \Gamma(\alpha+1) \tau\left(x_{0}\right) .
$$

Решение задачи Дирихле (6) для уравнения (9) представимо в виде

$$
\tau(x)=-\lambda \Gamma(\alpha+1) \tau\left(x_{0}\right) \int_{0}^{r} G(x, \xi) d \xi+G_{\xi}(x, r) \widetilde{\varphi}_{r}-G_{\xi}(x, 0) \widetilde{\varphi}_{0},
$$

где $G(x, \xi)$ - функция Грина задачи $(9),(6)$, имеющая вид

$$
G(x, \xi)= \begin{cases}\frac{\exp (k[\xi-x]) \operatorname{sh}(\mu \xi) \operatorname{sh}(\mu[x-r])}{\mu \operatorname{sh}(\mu r)}, & 0 \leqslant \xi \leqslant x, \\ \frac{\exp (k[\xi-x]) \operatorname{sh}(\mu x) \operatorname{sh}(\mu[\xi-r])}{\mu \operatorname{sh}(\mu r)}, & x \leqslant \xi \leqslant r,\end{cases}
$$

где $\mu=\sqrt{\Gamma^{2}(\alpha+1)-4 c \Gamma(\alpha+1)} / 2,2 k=\Gamma(\alpha+1), \mu r \neq \pi n i, n=1,2, \ldots, i-$ мнимая единица. Здесь надо отметить, что $G(x, \xi)$ является действительнозначной функцией, зависящей от комплексного параметра $\mu$.

При $x \rightarrow x_{0}$ из (10) получим

$$
\tau\left(x_{0}\right)=\frac{G_{\xi}\left(x_{0}, 0\right) \widetilde{\varphi_{0}}-G_{\xi}\left(x_{0}, r\right) \widetilde{\varphi_{r}}}{1+\lambda \Gamma(\alpha+1) \int_{0}^{r} G\left(x_{0}, \xi\right) d \xi}
$$

при выполнении условия

$$
1+\lambda \Gamma(\alpha+1) \int_{0}^{r} G\left(x_{0}, \xi\right) d \xi \neq 0 .
$$

Заметим, что условие (12) непусто; например при $c \leqslant 0, \lambda \leqslant 0$ оно всегда выполняется.

Таким образом, после того, как $\tau\left(x_{0}\right)$ найдено по формуле $(11), \tau(x)$ полностью определяется формулой (10), причем $\left.\tau(x) \in C[0, r] \cap C^{2}\right] 0, r[$. 
Решение задачи (1)-(4) в области гиперболичности $\Omega^{-}$выпишем так же, как и в [15]. Решение задачи Коши для уравнения (1) в $\Omega^{-}$имеет вид

$$
u(x, y)=\tau(x-y) e^{-c y}-\lambda \int_{0}^{y} u\left(x_{0}, \eta\right) e^{c(\eta-y)} d \eta .
$$

При $x \rightarrow x_{0}$ из (13) получим интегральное уравнение Вольтерра второго рода относительно функции $u\left(x_{0}, y\right)$ :

$$
u\left(x_{0}, y\right)+\lambda \int_{0}^{y} e^{c(\eta-y)} u\left(x_{0}, \eta\right) d \eta=e^{-c y} \tau\left(x_{0}-y\right),
$$

единственное решение которого задается формулой

$$
u\left(x_{0}, y\right)=e^{-c y}\left[\tau\left(x_{0}-y\right)-\lambda \int_{0}^{y} e^{\lambda(\eta-y)} \tau\left(x_{0}-\eta\right) d \eta\right] .
$$

Подставляя (10) и (14) в (13), получим решение задачи (1)-(4) в $\Omega^{-}$в явном виде.

Решение первой краевой задачи $(2),(5)$ для уравнения (1) в параболической части области $\Omega^{+}$ задается следующей формулой (см. [8]):

$$
u(x, y)=\left.\int_{0}^{y} \frac{\partial G_{1}}{\partial \xi}\right|_{\xi=0} \varphi_{0}(\eta) d \eta-\left.\int_{0}^{y} \frac{\partial G_{1}}{\partial \xi}\right|_{\xi=r} \varphi_{r}(\eta) d \eta+\int_{0}^{r} G_{1}(x, y ; \xi, 0) \tau(\xi) d \xi,
$$

где

$$
\begin{gathered}
G_{1}=G_{1}(x, y ; \xi, \eta)=\frac{\Gamma(\alpha)}{2}(y-\eta)^{\delta-1} \sum_{n=-\infty}^{\infty}\left[e_{1, \delta}^{1, \delta}\left(-\frac{|x-\xi+2 n|}{(y-\eta)^{\delta}}\right)\right]-\left[e_{1, \delta}^{1, \delta}\left(-\frac{|x+\xi+2 n|}{(y-\eta)^{\delta}}\right)\right], \\
\delta=\frac{\alpha}{2}, \quad e_{a, b}^{c, d}(z)=\sum_{k=0}^{\infty} \frac{z^{k}}{\Gamma(c+k a) \Gamma(d-b k)}-\text { функция Райта и } a>b \text { (см. [8]). }
\end{gathered}
$$

Теорема 1. Если функиии $y^{1-\alpha} \varphi_{0}(y), y^{1-\alpha} \varphi_{r}(y)$ принадлежсат классу $C\left(\bar{\Omega}^{+}\right)$и выполняется условие (12), то задача (1)-(4) имеет единственное решение, которое в $\Omega^{-}$задается формулой (13), а в $\Omega^{+}-$формулой (15).

\section{СПИСОК ЛИТЕРАТУРЫ}

1. Amтаев A.X. О некоторых задачах для нагруженного дифференциального уравнения в частных производных первого порядка// Вестн. КРАУНЦ. Физ.-мат. науки. - 2016. - 4-1 (16). - С. 9-14.

2. Геккиева C. X. Краевые задачи для нагруженных параболических уравнений с дробной производной по времени/ Дисс. на соиск. уч. степ. канд. физ.-мат. наук - Нальчик: НИИ ПМА КБНЦ РАН, 2003.

3. Геккиева С. Х. Смешанные краевые задачи для нагруженного диффузионно-волнового уравнения// Науч. вед. Белгород. гос. ун-та. Сер. мат. физ. - 2016. - 42, № 1 (12) 6 (227). - С. 32-35.

4. Дженалиев М. Т., Рамазанов М. И. Нагруженные уравнения как возмущения дифференциальных уравнений. - Алматы, 2010.

5. Нахушев А. М. Уравнения математической биологии. - М.: Высшая школа, 1995.

6. Нахушев А. М. Дробное исчисление и его применение. - М.: Физматлит, 2003.

7. Нахушев A. М. Нагруженные уравнения и их применения. - М.: Наука, 2012.

8. Псху А. В. Решение первой краевой задачи для уравнения диффузии дробного порядка// Диффер. уравн. - 2003. - 39, № 9. - С. 1286-1289.

9. Репин О. А. Краевая задача для дифференциального уравнения с частной дробной производной Римана-Лиувилля// Уфимск. мат. ж. - 2015. - 7, № 3. - С. 70-75.

10. Тарасенко А. В. О разрешимости нелокальной задачи для нагруженного параболо-гиперболического уравнения// Изв. вузов. Мат. - 2018. - 3. - С. 62-69. 
11. Тарасенко А. В., Егорова И. П. О нелокальной задаче с дробной производной Римана-Лиувилля для уравнения смешанного типа// Вестн. Самарск. гос. техн. ун-та. Сер. физ.-мат. науки. - 2017. - 21, № $1 .-$ C. $112-121$.

12. Хубиев K. У. Аналог задачи Трикоми для нагруженного уравнения гиперболо-параболического типа с дробной производной при нагрузке// Докл. Адыгск. (Черкесск.) Междунар. акад. наук. - 2015. 17, № 3. - С. 54-59.

13. Хубиев K. У. Внутреннекраевая задача для уравнения гиперболо-параболического типа с оператором дробной диффузии// Науч. вед. Белгород. гос. ун-та. Сер. мат. физ. - 2017. — 49, № 27 (276). С. $52-56$.

14. Хубиев K. У. Задачи со смещением для нагруженного уравнения гиперболо-параболического типа с оператором дробной диффузии// Вестн. Удмуртск. ун-та. Мат. Мех. Компьют. науки. - 2018. - 28, № 1. - C. $82-90$.

15. Хубиев К. У. Краевая задача для нагруженного уравнения гиперболо-параболического типа с вырождением порядка в области его гиперболичности// Итоги науки и техн. Совр. мат. прилож. Тематич. обзоры. - 2018. - 149. - C. 113-117.

16. Abdullaev O. Kh. On a problem for the loaded mixed type equation with fractional derivative// Bectн. КРАУНЦ. Физ.-мат. науки. — 2016. - 1 (12). — P. 7-14.

17. Abdullaev O. Kh., Sadarangani K. B. Nonlocal problem with integral gluing conditions for loaded mixed type equations involving the Caputo fractional derivate// Electron. J. Differ. Equations. — 2016. — 164. - P. 1-10.

18. Attaev A. Kh. The Cauchy problem for for the McKendrick-von Foerster loaded equation// Int. J. Pure Appl. Math. - 2017. — 113, № 4. - P. 47-52.

19. Baltaeva $U$. Solvability of the analogs of the problem Tricomi for the mixed type loaded equations with parabolic-hyperbolic operators// Boundary-Value Problems. — 2014. — 1. - P. 211-222.

20. Islomov B., Ochilova N. K. About a problem for the degenerating mixed type equation fractional derivative// Вестн. КРАУНЦ. Физ.-мат. науки. — 2017. — 1 (17). — Р. 22-32.

21. Kadirkulov B. J. Boundary problems for mixed parabolic-hyperbolic equations with two lines of changing type and fractional derivative// Electron. J. Differ. Equations. - 2014. - 57. — P. 1-7.

22. Karimov E. T., Akhatov J. S. A boundary problem with integral gluing condition for a parabolic-hyperbolic equation involving the Caputo fractional derivative// Electron. J. Differ. Equations. — 2014. — 14. P. 1-6.

Хубиев Казбек Узеирович

Институт прикладной математики и автоматизации,

Кабардино-Балкарский научный центр Российской академии наук, Нальчик

E-mail: khubiev_math@mail.ru 